\title{
Closed-loop updating of wind turbine models using blade pitch excitation
}

\author{
Bo M. Schwarz-Wolf ${ }^{\mathrm{a}, \mathrm{b}}$, Martin D. Ulriksen ${ }^{\mathrm{c}}$, Dawid Augustyn ${ }^{\mathrm{a}, \mathrm{b}}$, Martin S. Jensen ${ }^{\mathrm{b}}$, Ronnie R. Pedersen ${ }^{\mathrm{b}}$ \\ ${ }^{a}$ Department of the Built Environment, Aalborg University, Denmark \\ ${ }^{b}$ Ramboll Energy, Esbjerg, Denmark \\ ${ }^{c}$ Department of Energy Technology, Aalborg University, Denmark
}

\begin{abstract}
Structural monitoring of wind turbines often involves the use of a numerical model, which must be regularly updated to yield an adequate representation of the governing dynamics. The model updating can be cast as an inverse problem, in which selected model parameters are estimated by minimizing the discrepancy between experimental target poles and model-predicted ones. An issue that may prevail in this setting is that the problem will be ill-posed, because the number of model parameters to be updated exceeds the number of target poles. The noted issue can be remedied by a virtual implementation of output feedback, which allows for computation of multiple closed-loop (CL) eigenstructures through non-linear transformations of the open-loop transfer matrix. The present paper offers an application study, in which virtual output feedback is used for updating numerical wind turbine models based on CL system poles. In particular, we explore the feasibility of employing the excitation stemming from the blade pitch control system as the input in the open-loop input-output realization required in the virtual output feedback. The methodological concept is outlined, and subsequently the applicability of the procedure is tested numerically in the context of simulations with a finite element model of a parked wind turbine. The preliminary findings suggest that procedures for customizing the pitch excitation to yield sufficient system excitation are required for the CL model updating to be feasible.
\end{abstract}

Keywords: Structural health monitoring, Model updating, Output feedback, Wind turbines, Jacket substructures

\section{Introduction}

Offshore wind turbine systems operate under varying environmental and operational conditions, which are governed by, among other parameters, the wind, the waves, the temperature, and the control system. The shifts in these environmental and operational parameters (EOPs) render the structural properties time-variant and promote structural integrity degradation [1, 2], thus regular integrity assessments are required to ensure that the wind turbines operate adequately. A particular way of conducting the assessments is to use vibrationbased structural health monitoring (VSHM) techniques, in which the structural condition is inferred on the basis of processed vibration measurements [3]. Numerous VSHM techniques have been suggested for different levels of VSHM of wind turbine systems; covering damage detection [4], localization [5], quantification [6], and prognosis [7].

Numerical models are widely used in damage localization, assessment, and prognosis of wind turbines $[8,9]$. In order to ensure that the models provide an adequate representation of the governing system dynam- ics, they must be regularly calibrated through model updating [10]. Different studies have shown how indirect model updating - in which a subset of model parameters are estimated in an inverse problem setting - can allow for efficient calibration of numerical models of wind turbines and subsystems hereof [11, 12, 13, 14].

Indirect model updating aims to minimize the discrepancy between experimental target features and the corresponding model-predicted features by updating the selected model parameters. Let $\delta \lambda \in \mathbb{C}^{p}$ denote the discrepancy between the $p$ experimental and modelpredicted features and let $\boldsymbol{\theta} \in \mathbb{R}^{q}$ be the $q$ model parameters to be updated, then

$$
\delta \boldsymbol{\lambda} \approx \mathbf{J} \delta \boldsymbol{\theta},
$$

where $\mathbf{J} \in \mathbb{C}^{p \times q}$ is the Jacobian matrix containing the sensitivities of $\lambda$ with respect to $\boldsymbol{\theta}$. A commonly adopted approach in structural model updating is to calibrate a finite element model by matching system poles from the model with target poles estimated from experiments [10]. When using this setting for updating wind turbine models, a likely scenario is that problem (1) will 
be ill-posed, because the number of updating parameters, $q$, exceeds the number of identifiable poles, $p$.

The ill-posedness of problem (1) can be resolved using output feedback to design multiple closed-loop systems and hence target features $[15,16,17]$. To implement the feedback physically, the system input needs to be controllable, which will be infeasible in some applications. Instead, a signal processing-based implementation of the feedback can, as outlined in [18, 19, 20], be attained from available open-loop (OL) input-output realizations. As such, this implementation, which is referred to as virtual output feedback, eliminates the practical issues associated with closed-loop (CL) testing (including the physical constraints on the gain) and removes the constraint of CL system stability.

The present paper addresses the use of virtual output feedback for CL updating of finite element models of offshore wind turbine systems in parked conditions. In particular, we explore the feasibility of using excitation from the blade pitch control system as deterministic input in the virtual output feedback during parked conditions. Here, the pitch control system refers to the technology that is used to control the angle of the wind turbine blades in order to satisfy specific operational constraints governed by the wind speed, power output, and so forth. As such, the proposed procedure does not require any additions to the control system. This study is based on a numerical model of an $8 \mathrm{MW}$ offshore wind turbine system, which is introduced to stiffness-related perturbations that emulate common structural damage in wind turbine substructures.

The paper is organized as follows. In Section 2, the methodological principles of output feedback-based model updating are outlined. Section 3 describes the case study setup, including the use of pitch excitation input, while Section 4 presents the appertaining model updating results. The paper closes with some concluding remarks in Section 5.

\section{Methodology}

We consider a linear, time-invariant system of order $2 n$ with input $\mathbf{u}(k) \in \mathbb{R}^{r}$ and output $\mathbf{y}(k) \in \mathbb{R}^{m}$ at time instant $k$. The discrete-time state-space formulation of the system is

$$
\begin{aligned}
\mathbf{x}(k+1) & =\mathbf{A}_{d} \mathbf{x}(k)+\mathbf{B}_{d} \mathbf{u}(k), \\
\mathbf{y}(k) & =\mathbf{C}_{d} \mathbf{x}(k),
\end{aligned}
$$

where $\mathbf{A}_{d} \in \mathbb{R}^{2 n \times 2 n}, \mathbf{B}_{d} \in \mathbb{R}^{2 n \times r}$, and $\mathbf{C}_{d} \in \mathbb{R}^{m \times 2 n}$ are the state, input, and output matrices. In system (2), it is assumed that the direct transmission term is zero or subtracted from the output. Let system (2) operate under static output feedback given as

$$
\mathbf{u}(k)=-\mathbf{G y}(k)+\mathbf{v}(k),
$$

in which $\mathbf{G} \in \mathbb{R}^{r \times m}$ is the feedback gain and $\mathbf{v}(k) \in \mathbb{R}^{r}$ is a disturbance vector. By inserting (3) into system (2), we get the CL state equation

$$
\mathbf{x}(k+1)=\left(\mathbf{A}_{d}-\mathbf{B}_{d} \mathbf{G} \mathbf{C}_{d}\right) \mathbf{x}(k)+\mathbf{B}_{d} \mathbf{v}(k)
$$

with

$$
\overline{\mathbf{A}}=\mathbf{A}_{d}-\mathbf{B}_{d} \mathbf{G C}_{d}
$$

being the CL state matrix.

\subsection{Virtual output feedback}

The virtual implementation of output feedback follows from OL input-output realizations of system (2). In particular, let

$$
\mathbf{H}(z)=\mathbf{C}_{d}\left(z \mathbf{I}-\mathbf{A}_{d}\right)^{-1} \mathbf{B}_{d}
$$

be the OL transfer matrix of system (2), then, with feedback law (3), we establish

$$
\mathbf{y}(z)=\mathbf{H}(z)(-\mathbf{G y}(z)+\mathbf{v}(k)) .
$$

Hereby, the CL transfer matrix can be found as

$$
\overline{\mathbf{H}}(z)=(\mathbf{I}+\mathbf{H}(z) \mathbf{G})^{-1} \mathbf{H}(z),
$$

which implies that multiple CL systems can be computed based on a single OL input-output realization by
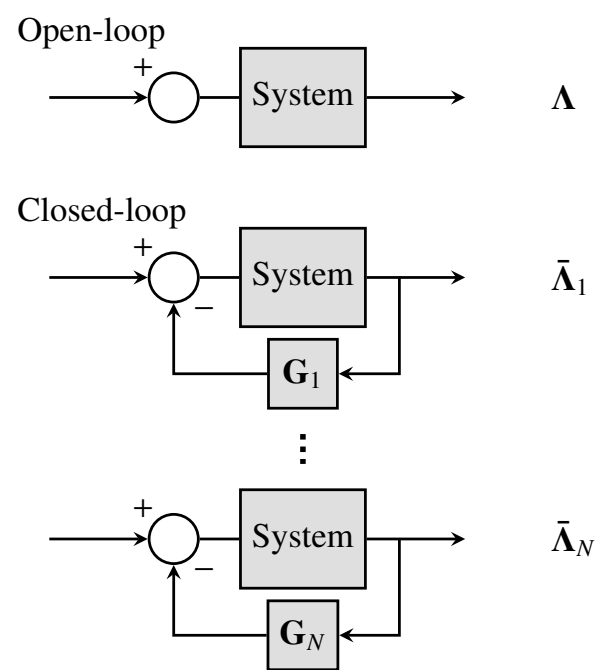

Figure 1: The concept of virtual output feedback with a total of $N$ CL systems based on a single OL input-output system realization. 
plugging in different gains, $\mathbf{G}_{j} \in \mathbb{R}^{r \times m}$. This concept is illustrated in Figure 1, where $\boldsymbol{\Lambda} \in \mathbb{C}^{2 n}$ is the full set of OL system poles while $\bar{\Lambda}_{j} \in \mathbb{C}^{2 n}$ contains the poles of the CL system formed using $\mathbf{G}_{j}$.

\subsection{Model updating formulation and gain design}

In practice, only a subset of $\boldsymbol{\Lambda}$ will be identifiable. To increase the dimensionality of the target feature vector, one can simply expand it with poles from $\overline{\boldsymbol{\Lambda}}_{j}$ (for $j=1, \ldots, N)$ until the inverse problem to be solved is (over)-determined with $p \geq q$. With the expanded target feature vector, the model updating can be conducted in full analogy to the conventional OL inverse problem formulations [10]. In the present study, the optimal model parameter set, $\hat{\boldsymbol{\theta}}$, is estimated as

$$
\hat{\boldsymbol{\theta}}=\underset{\boldsymbol{\theta} \in \mathbb{R}^{q}}{\arg \min }\left(\delta \boldsymbol{\lambda}^{T} \delta \boldsymbol{\lambda}\right),
$$

where $\delta \boldsymbol{\lambda}$ is specified as the difference between the experimental target features, $\lambda_{\mathcal{T}}$, and the model-predicted features, $\lambda_{\mathcal{M}(\boldsymbol{\theta})}$.

The $N$ gains to be used in the updating can be designed using different approaches [18, 21, 22, 23]. Here, we employ a gain design aiming to decrease the condition number of the Jacobian matrix in (1). Let

$$
\boldsymbol{G}=\left[\begin{array}{llll}
\mathbf{G}_{1}^{T} & \mathbf{G}_{2}^{T} & \ldots & \mathbf{G}_{N}^{T}
\end{array}\right]^{T} \in \mathbb{R}^{N r \times m}
$$

be the compound gain matrix, then

$$
\hat{\mathcal{G}}=\underset{\mathcal{G} \in \mathbb{R}^{N r \times m}}{\arg \min } \mathcal{K}(\mathbf{J})
$$

with $\mathcal{K}$ denoting the condition number operator. Worth of explicit note is that the gain design, for necessary practicality, is conducted on the basis of a model of the structural reference state, which does not take into account the current realization of the system parameters.

\section{Case study setup}

The offshore wind turbine model used in the numerical example is shown in Figure 2. The system, which is depicted without its rotor, consists of a three-legged jacket substructure with four brace levels connected to the soil with piles and an $8 \mathrm{MW}$ turbine. Details about the modeling, including dimensions, soil conditions, and damping properties, can be found in [24].

We use the terms nominal model and simulation model to refer to, respectively, the model to be updated and the model used to simulate experiments. The two models are shown in Figure 3, and it is noted that they

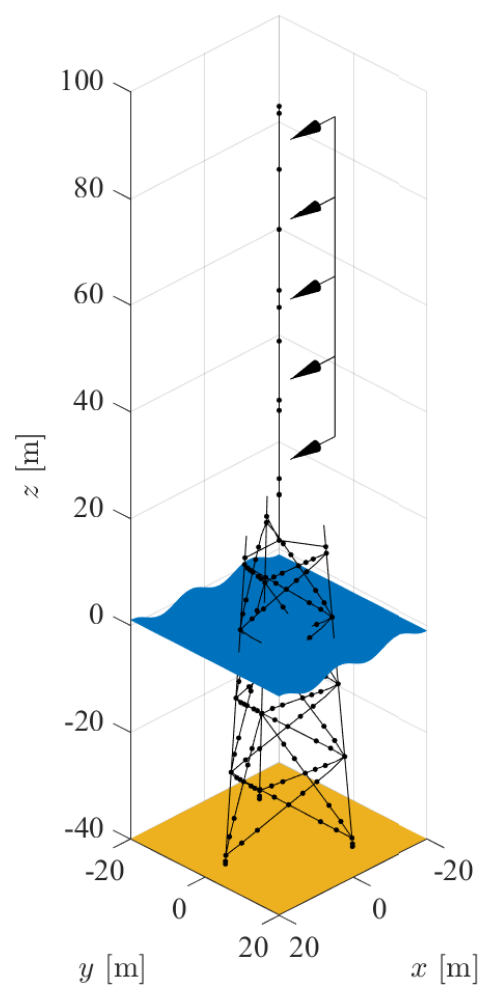

Figure 2: Wind turbine model used in the case study. The arrows indicate the wind load direction, which is co-directional with the waves.

both have 1200 degrees of freedom (DOF). The models have identical mass and damping matrices, denoted $\mathbf{M}, \mathbf{C} \in \mathbb{R}^{1200 \times 1200}$, while the stiffness, as outlined in Subsection 3.1, differs. The nominal model-whose stiffness, $\mathbf{K} \in \mathbb{R}^{1200 \times 1200}$, is to be updated-provides the model-predicted features, $\lambda_{\mathcal{M}(\boldsymbol{\theta})}$. Additionally, this model is used for designing the gains in accordance with the procedure outlined in Subsection 2.2.

In the simulations, the turbine is, as specified in Table 1 , in a parked condition with the impact from the wind and waves at a minimum. Hereby, the time-variant

Table 1: Load case used in the case study.

\begin{tabular}{ll}
\hline Parameter & Value \\
\hline Turbine state & Parked \\
Wind speed, $U[\mathrm{~m} / \mathrm{s}]$ & 4 \\
Wind profile & NWP* \\
Wave height, $H_{s}[\mathrm{~m}]$ & 0.45 \\
Wave period, $T_{p}[\mathrm{~s}]$ & 3.76 \\
Wind and wave direction & $\mathrm{N}^{* *}$ \\
\hline \multicolumn{2}{c}{${ }^{*}$ Normal wind profile (NWP) } \\
\multicolumn{2}{c}{${ }^{* *}$ North (N) }
\end{tabular}




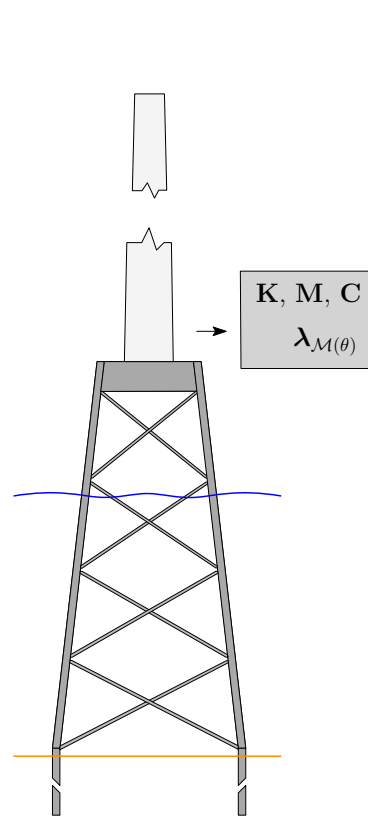

(a) Nominal model.

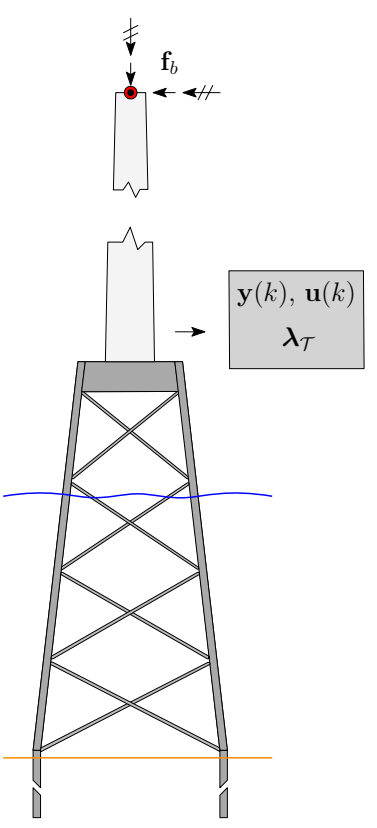

(b) Simulation model.
Figure 3: Models used in the case study. The load vector, $\mathbf{f}_{b}$, is explained in Subsection 3.2.

and non-linear effects induced by rotor operations are avoided, and the pitch excitation can be tailored to govern a part of the output. Each simulated OL input-output realization has a duration of $600 \mathrm{~s}$ and a time increment of $0.01 \mathrm{~s}$, hence totaling $N_{S}=60001$ sample points. From the realizations, the state-space system matrices are estimated as $\tilde{\mathbf{A}}_{d}, \tilde{\mathbf{B}}_{d}$, and $\tilde{\mathbf{C}}_{d}$ such that the OL target poles can be extracted as the eigenvalues of $\tilde{\mathbf{A}}_{d}$. The CL target poles are computed by plugging $\tilde{\mathbf{A}}_{d}, \tilde{\mathbf{B}}_{d}$, and $\tilde{\mathbf{C}}_{d}$ into (5) with a gain from the compound gain in (10). These estimated OL and CL poles compose the target features, $\lambda_{\mathcal{T}}$. Subsections 3.1 to 3.3 provide further details on the simulation model.

\subsection{Input, output, and damage locations}

To accommodate the constraints imposed by practicalities, it is chosen to place all output sensors in dry and accessible locations on the turbine. The sensors are taken as displacement sensors, which are distributed as shown in Figure 4. It is noted that at each output location, both $x$ - and $y$-directional displacements are captured, thus the output vector is $\mathbf{y}(k) \in \mathbb{R}^{8}$ for $k=0, \ldots, 60001$. The selected sensor distribution implies, as elaborated in [24], that the "local" brace modes of the system are not identifiable.

The loads used as deterministic input in the model updating are computed based on the load iteration pro-

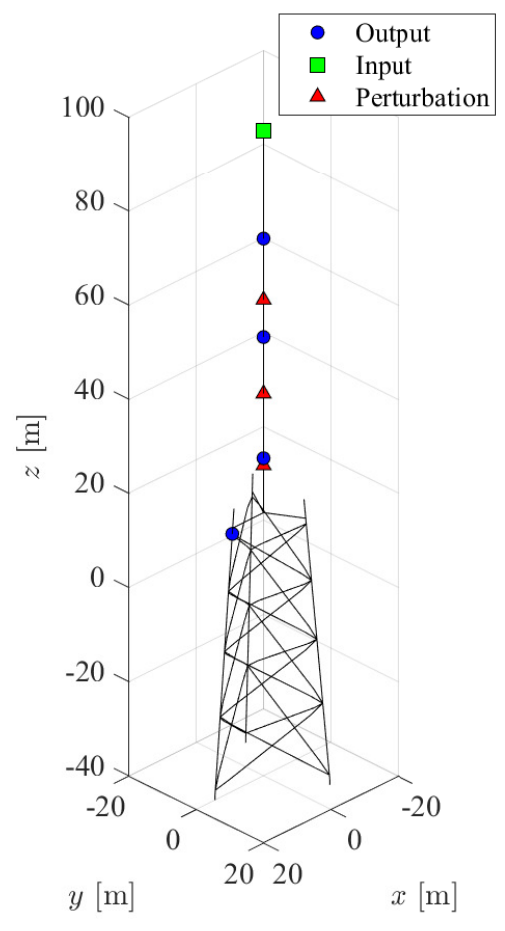

Figure 4: Input, output, and damage/stiffness perturbation locations.

cedure outlined in Subsection 3.2. The result is a set of interface loads, composed of $x$ - and $y$-directional forces and moments around the $x$ - and $y$-axes, at the location marked in Figure 4. Therefore, we get the input vector $\mathbf{u}(k) \in \mathbb{R}^{4}$ for $k=0, \ldots, 60001$. In practice, the input should be taken as the pitch loads acting in the rotor, which are either to be measured directly or estimated based on the available pitch angle time histories. The procedure selected in this study is thus merely for computational convenience.

The simulation model is, compared to the nominal model, perturbed by local stiffness changes at the locations indicated in Figure 4. With the first perturbation being the lowermost and the last the topmost, the perturbation vector is set to $[-20 \% 0-15 \%]$. The perturbations are introduced in three beam elements, which each has a length of approximately $3 \mathrm{~m}$ and is placed around a bolted connection in the tower; with the lowermost perturbation being at the bolted interface point between the tower and the jacket.

\subsection{Load iteration and response simulation}

The load iteration setup [25] depicted in Figure 5 is used to produce the required structural load input and 


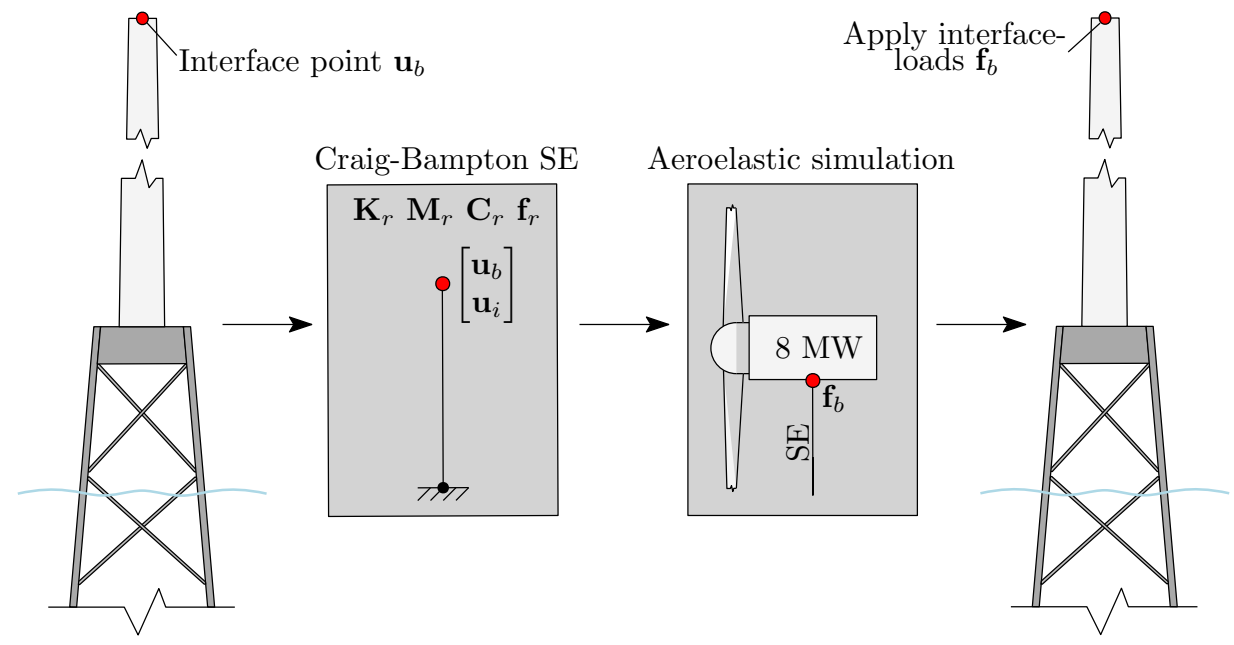

Figure 5: Load iteration used in input-output realizations.

response output. The setup includes the following four steps:

1. Initial Ramboll offshore structural analysis (ROSA) [26] model containing a wave model.

2. Craig-Bampton condensation [27] of the ROSA model and wave loads to generate a superelement (SE) with reduced mass, damping, stiffness, and loading $\left(\mathbf{M}_{r}, \mathbf{C}_{r}, \mathbf{K}_{r}\right.$, and $\left.\mathbf{f}_{r}\right)$ used in the subsequent aeroelastic simulation.

3. Aeroelastic simulation in LACflex [28], which computes the interface loads, $\mathbf{f}_{b}$.

4. Load recovery run in ROSA, with turbine loads from LACflex applied to the interface DOF, $\mathbf{u}_{b}$.

The last step of the load iteration, namely, the load recovery run, computes the structural response of the jacket and tower. Here, it is afterwards possible to extract displacements, stresses, and so forth.

\subsection{Pitch excitation}

The interface loads from the pitch excitation are used as input in the OL system identification, thus $\mathbf{u}(k)=$

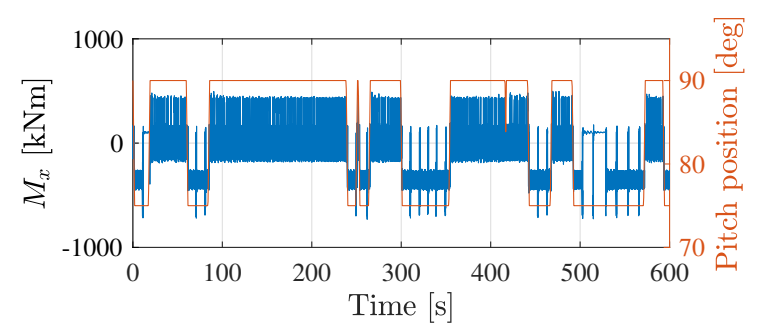

Figure 6: Bending moment $M_{x}$ at the interface point during pitching. $\mathbf{f}_{b}(k)$. The pitching is programmed such that all three blades rotate between two specific pitch angles, namely, $90 \mathrm{deg}$ and $75 \mathrm{deg}$. In Figure 6, the time history of one of the pitch excitation-induced loads, namely, the bending moment around the $x$-axis, $M_{x}$, is plotted along with the blade pitch angle. As expected, the temporal evolution of the moment correlates with the blade pitching.

To quantify the impact of the pitch excitation on the structural response, we plot, with reference to Figure 4 , the displacements captured in the topmost sensor. The results are shown in Figure 7 for the system with and without the pitch excitation; with $y_{1}$ being
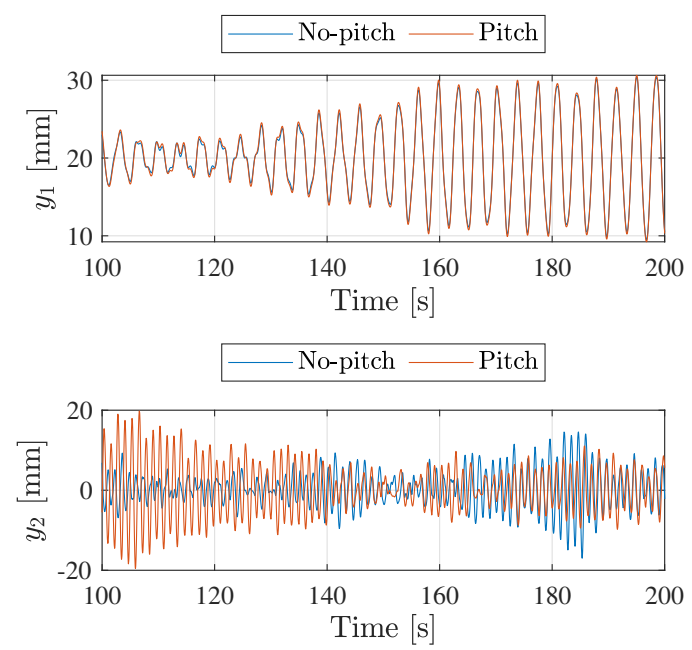

Figure 7: Structural displacement response at the topmost sensor (with reference to Figure 4) with and without pitch excitation. $y_{1}$ and $y_{2}$ are the $x$ - and $y$-directional displacements. 
Table 2: OL continuous-time poles of the nominal model, $\lambda_{\mathcal{M}}$, plus the exact, $\lambda_{\mathcal{T}}$, and estimated, $\tilde{\lambda}_{\mathcal{T}}$, poles of the simulation model.

\begin{tabular}{cccc}
\hline Pole & $\lambda_{\mathcal{M}}$ & $\lambda_{\mathcal{T}}$ & $\tilde{\lambda}_{\mathcal{T}}$ \\
\hline 1 & $-0.0430 \pm 5.0153 i$ & $-0.0431 \pm 4.9836 i$ & $-0.0427 \pm 4.9878 i$ \\
2 & $-0.0430 \pm 5.0153 i$ & $-0.0431 \pm 4.9837 i$ & \\
3 & $-0.1132 \pm 9.0915 i$ & $-0.1134 \pm 9.0529 i$ & $-0.1123 \pm 9.0845 i$ \\
4 & $-0.1132 \pm 9.0928 i$ & $-0.1134 \pm 9.0542 i$ & \\
\hline
\end{tabular}

the $x$-directional displacements and $y_{2}$ the $y$-directional displacements. Evidently, the pitching affects the $y$ directional response, while the $x$-directional response is not affected. The findings are a result of how the turbine loads are affected by the wind and wave direction, and how the turbine is rotated with respect to the global axis and jacket setup, as depicted in Figure 2.

\subsection{Updating features}

The input and output data is used to infer an estimate of the system and, as such, the employed target features through system identification. In this study, the datadriven subspace identification algorithm N4SID [29] is used under the precondition that the system is observable and controllable. The system identification yields consistent estimates of two poles of the wind turbine, which are presented in Table 2 along with the exact first and second bending mode poles from the simulation model and the nominal model. Evidently, the attained estimates match, with reasonable accuracy, the poles of the first two sets of bending modes. The corresponding exact poles from the nominal model have, as expected, higher frequencies since no perturbations are present.

\section{Results}

The updating is conducted in a setting where the three model parameters perturbed with $[-20 \% 0 \%-15 \%]$ in the simulation model are to be estimated in the nominal model. With three model parameters, the two estimated OL poles are insufficient to render the updating problem well-posed, so CL systems are formed to expand the target vector such $p \geq q$. We design three CL systems from each of which we extract the two CL poles that "correspond" to the two OL poles. The target feature is thus a mixture of two OL and six CL poles.

With the target vector discrepancy $\delta \lambda \in \mathbb{C}^{8}$, the three model parameters are estimated via minimization of

$$
\beta=\delta \lambda^{T} \delta \lambda
$$

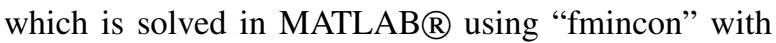
an interior-point algorithm [30]. The results for different initial configurations, $\delta \boldsymbol{\theta}_{0}$, are listed in Table 3 . As can be seen, the CL updating scheme fails to provide the correct perturbation configuration, as it, even when initializing the optimization from the true configuration, converges to smaller perturbations (in an absolute sense). The associated OL poles for this converged model configuration are presented in Table 4, where it can be appreciated that the frequency of the first pole is above the target value, while the frequency of the second pole is below the target value. It is noted that when conducting the updating with just the two OL poles, rendering the problem ill-posed, the results are $\left[\begin{array}{lll}-12 \% & -2.2 \% & 1.3 \%\end{array}\right]^{T}$ for $\delta \boldsymbol{\theta}_{0}=\left[\begin{array}{lll}0 & 0 & 0\end{array}\right]^{T}$ and $[-9.3 \% 1.3 \%-5.8 \%]^{T}$ for $\delta \boldsymbol{\theta}_{0}=[-20 \% 0 \%-15 \%]^{T}$.

The error in the estimate of the second OL pole, see Table 2, is a likely cause of the erroneous findings in Tables 3 and 4. Therefore, we conduct a new update of the nominal model based solely on the first OL pole and three "corresponding" CL modes. The appertaining results are listed in Table 5 for different initial configurations. When initializing the optimization from

Table 3: CL updating results using eight target features with $\delta \boldsymbol{\theta}$ in percentage. Note that 'a' is the initial evaluation of the cost function and ' $b$ ' the converged.

\begin{tabular}{|c|c|c|c|c|c|}
\hline Setup & $\delta \boldsymbol{\theta}_{0}^{T}$ & $\delta \hat{\theta}_{1}$ & $\delta \hat{\theta}_{2}$ & $\delta \hat{\theta}_{3}$ & $\beta\left(10^{-3}\right)$ \\
\hline $1 \mathrm{a}$ & {$\left[\begin{array}{lll}0 & 0 & 0\end{array}\right]$} & \multicolumn{3}{|c|}{ Initial evaluation } & 5.2 \\
\hline $1 b$ & {$\left[\begin{array}{lll}0 & 0 & 0\end{array}\right]$} & -13 & -16 & 8.0 & 0.63 \\
\hline $2 \mathrm{a}$ & {$\left[\begin{array}{lll}-20 & 0 & -15\end{array}\right]$} & \multicolumn{3}{|c|}{ Initial evaluation } & 2.8 \\
\hline $2 b$ & {$\left[\begin{array}{lll}-20 & 0 & -15\end{array}\right]$} & -14 & 3.2 & -3.9 & 0.70 \\
\hline
\end{tabular}

Table 4: Estimated target poles and poles of the nominal model updated with the converged solution in configuration $2 \mathrm{~b}$ in Table 3.

\begin{tabular}{cc}
\hline$\tilde{\lambda}_{\mathcal{T}}$ & $\lambda_{\mathcal{M}(\hat{\boldsymbol{\theta}})}$ \\
\hline$-0.0427 \pm 4.9878 i$ & $-0.0431 \pm 4.9978 i$ \\
$-0.1123 \pm 9.0845 i$ & $-0.1133 \pm 9.0744 i$ \\
\hline
\end{tabular}


Table 5: CL updating results using only the first OL pole and three "corresponding" CL poles with $\delta \boldsymbol{\theta}$ in percentage. Note that 'a' is the initial evaluation of the cost function and 'b' the converged.

\begin{tabular}{|c|c|c|c|c|c|}
\hline Setup & $\delta \boldsymbol{\theta}_{0}^{T}$ & $\delta \hat{\theta}_{1}$ & $\delta \hat{\theta}_{2}$ & $\delta \hat{\theta}_{3}$ & $\beta\left(10^{-4}\right)$ \\
\hline $1 \mathrm{a}$ & {$\left[\begin{array}{lll}0 & 0 & 0\end{array}\right]$} & \multicolumn{3}{|c|}{ Initial evaluation } & 36 \\
\hline $1 b$ & {$\left[\begin{array}{lll}0 & 0 & 0\end{array}\right]$} & -22 & -7.0 & -6.8 & 1.1 \\
\hline $2 \mathrm{a}$ & {$\left[\begin{array}{lll}-20 & 0 & -15\end{array}\right]$} & \multicolumn{3}{|c|}{ Initial evaluation } & 1.2 \\
\hline $2 b$ & {$\left[\begin{array}{lll}-20 & 0 & -15\end{array}\right]$} & -21 & -0.26 & -15 & 1.1 \\
\hline
\end{tabular}

$\delta \boldsymbol{\theta}_{0}=\left[\begin{array}{lll}0 & 0 & 0\end{array}\right]^{T}$, the three model parameters converge at values that do not coincide with the true values. In the case of $\delta \boldsymbol{\theta}_{0}=[-20 \% 0 \%-15 \%]^{T}$, the three model parameters converge in the vicinity of the theoretical values. The attained cost function is, however, equal to the one attained in setup 1, so although using only the first OL pole and the "corresponding" CL poles improves the results, there is still some clear room for improvement. It is contended that this can be achieved if the pitch excitation can be tailored trough input shaping [31, 32], such that more accurate estimates can be obtained of a subset of the OL poles.

\section{Conclusion}

The paper explores the feasibility of employing blade pitch-induced excitation as input in CL updating of numerical models of parked offshore wind turbines. The merit of the CL implementation is that it can be formulated such that the inverse problem to be solved in the model updating is well-posed. A case study with a numerical wind turbine system is presented, and although the current, preliminary findings do not appear particularly encouraging, it is contended that the encountered issues can be resolved by tailoring the delivered pitch excitation to allow for improved estimation of the system poles. This is a part of the ongoing research.

\section{References}

[1] C. C. Ciang, J.-R. Lee, H.-J. Bang, Structural health monitoring for a wind turbine system: a review of damage detection methods, Measurement Science and Technology 19 (2008) 122001. doi:10.1088/0957-0233/19/12/122001.

[2] J. F. Skov, M. D. Ulriksen, K. A. Dickow, P. H. Kirkegaard, L. Damkilde, On structural health monitoring of wind turbine blades, in: Damage Assessment of Structures X, volume 569 of Key Engineering Materials, 2013, pp. 628-635. doi:10.4028/www.scientific.net/KEM.569-570.628.

[3] C. R. Farrar, K. Worden, Structural health monitoring: A machine learning perspective, 1 ed., John Wiley \& Sons, Inc., 2013.
[4] G. Oliveira, F. Magalhães, A. Cunha, E. Caetano, Vibrationbased damage detection in a wind turbine using 1 year of data, Structural Control and Health Monitoring 25 (2018) e2238. doi: $10.1002 /$ stc. 2238

[5] M. D. Ulriksen, D. Tcherniak, L. M. Hansen, R. J. Johansen, L. Damkilde, L. Frøyd, In-situ damage localization for a wind turbine blade through outlier analysis of stochastic dynamic damage location vector-induced stress resultants, Structural Health Monitoring 16 (2017) 745-761. doi: $10.1177 / 1475921716681727$.

[6] A. Abouhnik, A. Albarbar, Wind turbine blades condition assessment based on vibration measurements and the level of an empirically decomposed feature, Energy Conversion and Management 64 (2012) 606-613. doi:10.1016/j.enconman.2012.06.008.

[7] A. Iliopoulos, W. Weijtjens, D. Van Hemelrijck, C. Devriendt, Fatigue assessment of offshore wind turbines on monopile foundations using multi-band modal expansion, Wind Energy 20 (2017) 1463-1479. doi:10.1002/we.2104.

[8] Y. Du, S. Zhou, X. Jing, Y. Peng, H. Wu, N. Kwok, Damage detection techniques for wind turbine blades: A review, Mechanical Systems and Signal Processing 141 (2020) 106445. doi:10.1016/j.ymssp.2019.106445.

[9] M. D. Ulriksen, Damage Localization for Structural Health Monitoring: An Exploration of Three New Vibration-based Schemes, Ph.D. thesis, Aalborg University, Aalborg, Denmark, 2018.

[10] M. I. Friswell, J. E. Mottershead, Finite Element Model Updating in Strucural Dynamics, Kluwer Academic Publishers, 1995.

[11] M. Luczak, S. Manzato, B. Peeters, K. Branner, P. Berring, M. Kahsin, Updating finite element model of a wind turbine blade section using experimental modal analysis results, Shock and Vibration 2014 (2014) 684786. doi:10.1155/2014/684786.

[12] B. Hofmeister, M. Bruns, R. Rolfes, Finite element model updating using deterministic optimisation: A global pattern search approach, Engineering Structures 195 (2019) 373-381. doi:10.1016/j.engstruct.2019.05.047.

[13] D. Augustyn, U. Smolka, U. T. Tygesen, M. D. Ulriksen, J. D. Sørensen, Data-driven model updating of an offshore wind jacket substructure, Applied Ocean Research 104 (2020) 102366. doi:10.1016/j.apor.2020.102366.

[14] Y. Xu, G. Nikitas, T. Zhang, Q. Han, M. Chryssanthopoulos, S. Bhattacharya, Y. Wang, Support condition monitoring of offshore wind turbines using model updating techniques, Structural Health Monitoring 19 (2020) 1017-1031. doi: $10.1177 / 1475921719875628$.

[15] L. J. Jiang, J. J. Tang, K. W. Wang, An optimal sensitivityenhancing feedback control approach via eigenstructure assignment for structural damage identification, Journal of Vibration and Acoustics 129 (2007) 771-783. doi:10.1115/1.2748476.

[16] K. D’Souza, B. I. Epureanu, Damage detection in nonlinear systems using optimal feedback auxiliary signals and system augmentations, Journal of Vibration and Acoustics 132 (2010) 021002-021002-9. doi:10.1115/1.4000839.

[17] D. Bernal, M. D. Ulriksen, Output feedback in the design of eigenstructures for enhanced sensitivity, Mechanical Systems and Signal Processing 112 (2018) 22-30. doi:10.1016/j.ymssp.2018.04.032.

[18] D. Bernal, Virtual output feedback in parameter estimation, Mechanical Systems and Signal Processing 142 (2020) 106786. doi:10.1016/j.ymssp.2020.106786.

[19] D. Bernal, M. D. Ulriksen, Virtual closed loop parameter estimation: a review, in: International Conference on Structural Engineering Dynamics (ICEDyn), 2019, pp. 8-14.

[20] M. S. Jensen, T. N. Hansen, M. D. Ulriksen, D. Bernal, Physi- 
cal and virtual implementation of closed-loop designs for model updating, in: Z. Jamaludin, M. Ali Mokhtar, M. Wahab (Eds.), Proceedings of the 13th International Conference on Damage Assessment of Structures, Lecture Notes in Mechanical Engineering, 2020, pp. 363-371. doi:10.1007/978-981-13-8331$1 \_25$.

[21] M. D. Ulriksen, D. Bernal, On the use of complex gains in virtual feedback for model updating, in: International Conference on Structural Engineering Dynamics (ICEDyn), 2019, pp. 1-7.

[22] M. D. Ulriksen, D. Bernal, On gain design in virtual output feedback for model updating, in: Z. Jamaludin, M. N. A. Mokhtar, M. A. Wahab (Eds.), Proceedings of the 13th International Conference on Damage Assessment of Structures, Lecture Notes in Mechanical Engineering, 2020, pp. 372-379. doi:10.1007/978981-13-8331-1_26.

[23] L. T. Hansen, T. Akselsen, U. Korkmaz, M. D. Ulriksen, A comparison of gain design criteria for closed-loop model updating, in: M. Papadrakakis, M. Fragiadakis, P. C. (Eds.), Proceedings of XI International Conference on Structural Dynamics, EURODYN 2020, 2020, pp. 4630-4637. doi:978-618-85072-2-7.

[24] D. Augustyn, R. R. Pedersen, U. T. Tygesen, M. D. Ulriksen, J. D. Sørensen, Feasibility of modal expansion for virtual sensing in offshore wind jacket substructures, Marine Structures (2020). (submitted).

[25] M. B. Nielsen, J. F. Jensen, C. Harper, L. S. Knudsen, R. R Pedersen, State-of-the-art framework for design of offshore wind jacket foundations, Steel Construction 12 (2019) 209-214 doi:10.1002/stco.201900016.

[26] Ramboll, Tech. rep., Rosap - Ramboll Offshore Structural Analysis Package, 2020. Version 54.

[27] R. D. C. et al, Concepts and Applications of Finite Element Analysis, 4. ed. ed., John Wiley and Sons, INC., 2002. ISBN 978-0-471-35605-9.

[28] Ramboll, Tech. rep., LACflex - aeroelastic simulation, 2020. Version 1.12.1.

[29] P. van Overschee, B. D. Moor, Subspace identification for linear systems, Kluwer Academic Publishers, 1996. ISBN 0-79239717-7.

[30] R. Byrd, J. Gilbert, J. Nocedal, A trust region method based on interior point techniques for nonlinear programming, Mathematical Programming 89 (2000) 149-185. doi:10.1007/PL00011391.

[31] T. Singh, G. R. Heppler, Shaped input control of a system with multiple modes, Journal of Dynamic Systems, Measurement, and Control 115 (1993) 341-347. doi:10.1115/1.2899108.

[32] M. D. Ulriksen, D. Bernal, L. Damkilde, Shaped input distributions for structural damage localization, Mechanical Systems and Signal Processing 110 (2018) 499-508. doi:10.1016/j.ymssp.2018.03.039. 